\title{
INFLUENCE OF MATERIAL NON-LINEARITY ON DELAMINATION IN MULTILAYERED THREE-POINT BENDING BEAMS
}

\author{
Victor Rizov ${ }^{1 *}$, Holm AltenbaCH ${ }^{2}$ \\ ${ }^{1}$ Department of Technical Mechanics, University of Architecture, Civil \\ Engineering and Geodesy, 1 Chr. Smirnensky blvd., 1046 Sofia, Bulgaria \\ ${ }^{2}$ Lehrstuhl für Technische Mechanik und Geschäftsführender \\ Leiter Institut für Mechanik G10/58, Fakultät für Maschinenbau, \\ Otto-von-Guericke-Universität Magdeburg, \\ Universitätsplatz 2, 39106 Magdeburg, Deutschland
}

[Received: 05 July 2019. Accepted: 21 October 2019]

doi: 10.7546/JTAM.50.20.01.07

\begin{abstract}
Delamination in multilayered three-point bending beam configurations is analyzed assuming non-linear behaviour of the material in each layer. For this purpose, an analytical approach is developed by using the RambergOsgood equation. A solution for the strain energy release rate is derived by considering the energy balance. The strain energy release rate is obtained also by analyzing the complementary strain energy for verification. The solution derived is valid for a beam made of an arbitrary number of adhesively bonded horizontal layers of different thicknesses and material properties. Besides, the delamination can be located arbitrary between layers. The solution is applied for parametric investigations of delamination behaviour. The effects of delamination position along the beam height, material non-linearity and delamination length on delamination behaviour are evaluated. The results obtained in the present study are benefit to structural design of multilayered three-point bending beams exhibiting material non-linearity.
\end{abstract}

KEY WORDS: delamination crack, material non-linearity, multilayered structure, three-point bending.

\section{INTRODUCTION}

The widespread use of multilayered materials in various structural members and components has been attempted mainly in order to reduce the structural weight [1-7]. Delamination, i.e separation of layers, is one of the most common failure modes in layered structures [8-19].

A solution to the strain energy release rate for delaminaton fracture in multilayered beam configurations has been derived in [11]. The delamination has been analyzed assuming linear-elastic behaviour of the multilayered material. The solution

\footnotetext{
${ }^{*}$ Corresponding author e-mail: V_RIZOV_FHE@UACG.BG
} 
derived has been applied for studying delamination fracture in both three-layered and four-layered linear-elastic beam structures. It has been found that the solution can be used successfully for studying delamination fracture behaviour of deposited metal layers.

Delamination fracture in different multilayered beam configurations has been studied in [18]. It has been assumed that the material in each layer of the beams has linear-elastic behaviour. Solutions to the strain energy release rate for the beams under consideration have been obtained. For this purpose, linear-elastic fracture mechanics has been used. The solutions have been applied to calculate the strain energy release rate for a given geometry and mechanical properties. It has been shown that the solutions obtained can be useful for evaluation of delamination fracture behaviour of multilayered linear-elastic beam structures.

Delamination fracture of multilayered beams exhibiting linear-elastic behaviour has been investigated in [19]. A vertical notch is introduced in the mid-span in order to generate conditions for delamination fracture of the multilayered beam configuration. A delamination crack is located arbitrary with respect to the mid-span. The delamination has been studied in terms of the strain energy release rate. Methods of linear-elastic fracture mechanics have been applied in order to analyze the strain energy release rate. The solution derived has been used to evaluate the effects of various geometrical and material parameters on the delamination fracture behaviour of the multilayered beam structure.

The literature review shows that delamination of multilayered beam structures has been investigated mainly assuming linear-elastic behaviour. Therefore, the present paper is concerned with a delamination analysis of a multilayered three-point bending beam (i.e., a simply supported beam loaded by one vertical force applied in the mid-span) assuming non-linear behaviour of the material in each layer. The material non-linearity is treated with the Ramberg-Osgood stress-strain relation. It should be mentioned that one of the basic problems when using the Ramberg-Osgood stressstrain relation is the fact that the stress can not be determined explicitly. In the present paper, an approach which solves this problem is developed. The delamination analysis developed in the present paper holds for multilayered beams made of layers of different homogeneous materials. Approaches for analyzing delamination fracture behaviour of multilayered beams whose layers are made of functionally graded materials are reported in $[20,21]$. The originality of the present paper in comparison with $[20,21]$ consists in the fact that the approach developed here does not necessitate expanding of the stress in series of Taylor. Thus, determination of the coefficients of the series of Taylor is avoided which makes the present approach more convenient for application. 


\section{Determination of the Strain EnERgy Release Rate}

The delamination crack in the multilayered three-point bending beam shown in Fig. 1 is analyzed. The beam cross-section is a rectangle of width $b$ and height $2 h$. The beam length is $2 l$. It is assumed that the beam is made of adhesively bonded horizontal layers of different thicknesses and material properties. The number of layers is arbitrary. A delamination crack of length $a$ is located arbitrary between layers. The thicknesses of the upper and lower crack arms are $h_{1}$ and $h_{2}$, respectively. The beam is loaded by one vertical force $F$ applied in the mid-span. Obviously, the lower crack arm is free of stresses (Fig. 1). It is assumed that the material ehxibits non-linear behaviour in each layer. The delamination is analyzed in terms of the strain energy release rate $G$. For this purpose, the energy balance is written as:

$$
F \delta w=\frac{\partial U}{\partial a} \delta a+G b \delta a,
$$

where $U$ is the strain energy cumulated in the beam, $\delta a$ is a small increase of the delamination length, $w$ is the vertical displacement of the aplication point of the force, $F$. From (1), $G$ is expressed as:

$$
G=\frac{F}{b} \frac{\partial w}{\partial a}-\frac{1}{b} \frac{\partial U}{\partial a} .
$$

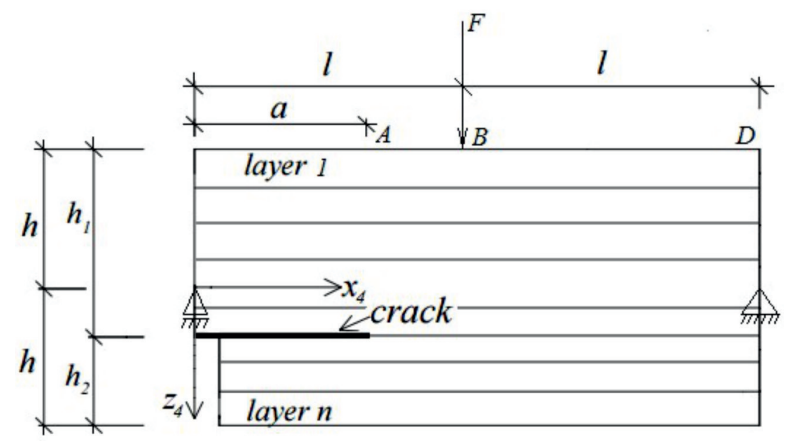

Fig. 1. Multilayered three-point bending beam with a delamination crack.

By using the integrals of Maxwell-Mohr, the vertical displacement of the aplication point of force $F$ is obtained as:

$$
w=\int_{0}^{a} \frac{1}{2} x_{4} \kappa_{1} d x_{4}+\int_{a}^{l} \frac{1}{2} x_{4} \kappa_{2} d x_{4}+\int_{l}^{2 l}\left(l-\frac{1}{2} x_{4}\right) \kappa_{3} d x_{4},
$$


where $\kappa_{1}$ is the curvature of the upper crack arm, $\kappa_{2}$ and $\kappa_{3}$ are the curvatures, respectively, of portions $A B$ and $B D$ of the un-cracked part of the beam, $x_{4}$-axis is shown in Fig. 1.

The following equations for equilibrium of the elementary forces in the crosssection of the upper crack arm are used in order to determine $\kappa_{1}$ :

$$
\begin{aligned}
& N_{1}=b \sum_{i=1}^{i=n_{U}} \int_{z_{1 i}}^{z_{1 i+1}} \sigma_{i} d z_{1}, \\
& M_{y_{1}}=b \sum_{i=1}^{i=n_{U}} \int_{z_{1 i}}^{z_{1 i+1}} \sigma_{i} z_{1} d z_{1},
\end{aligned}
$$

where $n_{U}$ is the number of layers in the upper crack arm, $z_{1 i}$ and $z_{1 i+1}$ are the coordinates, respectively, of the upper and lower surfaces of the $i$-th layer (Fig. 2), $\sigma_{i}$ is the distribution of the longitudinal normal stresses in the same layer, $N_{1}$ and $M_{y_{1}}$ are, respectively, the axial force and bending moment in the upper crack arm (apparently, $N_{1}=0$ and $M_{y_{1}}=F x_{4} / 2$ ).

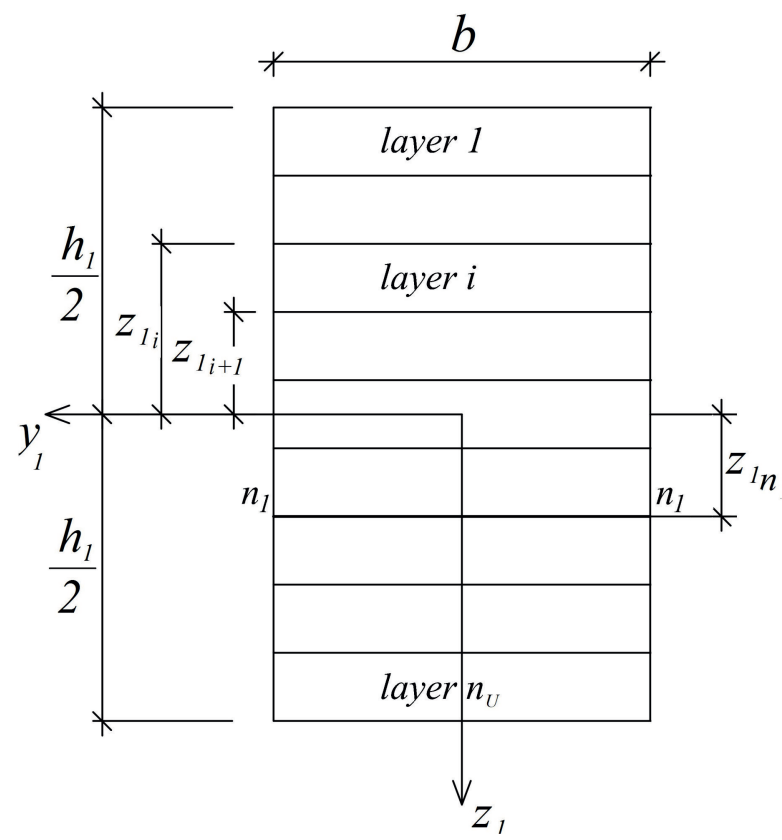

Fig. 2. Cross-section of the upper crack arm $\left(n_{1}-n_{1}\right.$ is the position of neutral axis). 
In the present paper, it is assumed that the material non-linearity can be described by the Ramberg-Osgood stress-strain relation:

$$
\varepsilon=\frac{\sigma_{i}}{E_{i}}+\left(\frac{\sigma_{i}}{H_{i}}\right)^{\frac{1}{m_{i}}},
$$

where $\varepsilon$ is the longitudinal strain, $E_{i}$ is the modulus of elasticity, $H_{i}$ and $m_{i}$ are material properties in the $i$-th layer. It should be noted that the first term in the righthand side of (6) describes the linear behaviour, while the second term models the material non-linearity. The distribution of $\varepsilon$ along the height of the cross-section is written as:

$$
\varepsilon=\kappa_{1}\left(z_{1}-z_{1 n_{1}}\right),
$$

since it is assumed validity of the Bernoulli's hypothesis for plane sections becouse the span to height ratio of the beam under consideration is large. In (7), $z_{1 n_{1}}$ is the coordinate of the neutral axis (it should be noted that the neutral axis $n_{1}-n_{1}$ shifts from the centroid becouse the beam is multilayered (Fig. 2)).

The stress $\sigma_{i}$ has to be expressed as a function of the coordinate $z_{1}$ in order to perform the integration in (4) and (5). However, it is obvious that $\sigma_{i}$ can not be determined explicitly form (6). Therefore, by combining of (6) and (7), one expresses $z_{1}$ and $d z_{1}$ as functions of $\sigma_{i}$ :

$$
\begin{aligned}
& z_{1}=\frac{1}{\kappa_{1}}\left[\frac{\sigma_{i}}{E_{i}}+\left(\frac{\sigma_{i}}{H_{i}}\right)^{\frac{1}{m_{i}}}\right]+z_{1 n_{1}}, \\
& d z_{1}=\frac{1}{\kappa_{1}}\left[\frac{1}{E_{i}}+\frac{\sigma_{i}^{\frac{1-m_{i}}{m_{i}}}}{m_{i} H_{i}^{\frac{1}{m_{i}}}}\right] d \sigma_{i} .
\end{aligned}
$$

By substituting of (8) and (9) in (4) and (5) and integrating in boundaries from $\sigma_{q_{i}}$ to $\sigma_{r_{i}}$, one obtains:

$$
\begin{aligned}
& N_{1}=\frac{b}{\kappa_{1}} \sum_{i=1}^{i=n_{U}}\left[\frac{\sigma_{r_{i}}^{2}}{2 E_{i}}+\frac{\sigma_{r_{i}}^{\frac{1+m_{i}}{m_{i}}}}{\left(1+m_{i}\right) H_{i}^{\frac{1}{m_{i}}}}-\frac{\sigma_{q_{i}}^{2}}{2 E_{i}}-\frac{\sigma_{q_{i}}^{\frac{1+m_{i}}{m_{i}}}}{\left(1+m_{i}\right) H_{i}^{\frac{1}{m_{i}}}}\right], \\
& M_{y_{1}}=\frac{b}{\kappa_{1}} \sum_{i=1}^{i=n_{U}}\left[\frac{\sigma_{r_{i}}^{3}-\sigma_{q_{i}}^{3}}{3 \kappa_{1} E_{i}^{2}}+\left(\sigma_{r_{i}}^{\frac{1+2 m_{i}}{m_{i}}}-\sigma_{q_{i}}^{\frac{1+2 m_{i}}{m_{i}}}\right) \frac{m_{i}+1}{\kappa_{1} E_{i} H_{i}^{\frac{1}{m_{i}}}\left(1+2 m_{i}\right)}\right. \\
& \left.+\frac{z_{1 n_{1}}\left(\sigma_{r_{i}}^{2}-\sigma_{q_{i}}^{2}\right)}{2 E_{i}}+\frac{\sigma_{r_{i}}^{\frac{2+m_{i}}{m_{i}}}-\sigma_{q_{i}}^{\frac{2+m_{i}}{m_{i}}}}{\kappa_{1} H_{i}^{\frac{2}{m_{i}}}\left(2+m_{i}\right)}+\frac{z_{1 n_{1}}\left(\sigma_{r_{i}}^{\frac{1+m_{i}}{m_{i}}}-\sigma_{q_{i}}^{\frac{1+m_{i}}{m_{i}}}\right)}{H_{i}^{\frac{1}{m_{i}}}\left(1+m_{i}\right)}\right],
\end{aligned}
$$


where $\sigma_{q_{i}}$ and $\sigma_{r_{i}}$ are the normal stresses, respectively, in the upper and lower surfaces of the $i$-th layer.

It should be mentioned that at $n_{U}=1, E_{i}=E$ and $H_{i} \rightarrow \infty$ equation (11) transforms in:

$$
\kappa_{1}=\frac{12 M_{y_{1}}}{E b h_{1}^{3}},
$$

which matches exactly the formula for curvtarure of a linear-elastic homogeneous beam. This fact is an indication for consistency of (11) since at $H_{i} \rightarrow \infty$ the Ramberg-Osgood equation transforms into the Hooke's law.

The number of un-knowns in equations (10) and (11) is $2 n_{U}+2$, i.e. $\kappa_{1}, z_{1 n_{1}}$, $\sigma_{q_{i}}$ and $\sigma_{r_{i}}$, where $i=1,2, \ldots, n_{U}$. By combining of (6) and (7), one derives $2 n_{U}$ aditional equations:

$$
\begin{aligned}
& \kappa_{1}\left(z_{1 i}-z_{1 n_{1}}\right)=\frac{\sigma_{q_{i}}}{E_{i}}+\left(\frac{\sigma_{q_{i}}}{H_{i}}\right)^{\frac{1}{m_{i}}}, \\
& \kappa_{1}\left(z_{1 i+1}-z_{1 n_{1}}\right)=\frac{\sigma_{r_{i}}}{E_{i}}+\left(\frac{\sigma_{r_{i}}}{H_{i}}\right)^{\frac{1}{m_{i}}},
\end{aligned}
$$

where $i=1,2, \ldots, n_{U}$. Equations (10), (11), (13) and (14) should be solved with respect to $\kappa_{1}, z_{1 n_{1}}, \sigma_{q_{i}}$ and $\sigma_{r_{i}}$, where $i=1,2, \ldots, n_{U}$, by using the MatLab computer progtam. It should be mentioned that by using equations (10), (11), (13) and (14), one can determine $\kappa_{1}, z_{1 n_{1}}, \sigma_{q_{i}}$ and $\sigma_{r_{i}}$, where $i=1,2, \ldots, n_{U}$, for any cross-section of the upper crack arm. Equations (10), (11), (13) and (14) can also be applied to obtaine $\kappa_{2}$, the coordinate of the neutral axis $z_{2 n_{2}}$ and the normal stresses $\sigma_{A B q_{i}}$ and $\sigma_{B D r_{i}}$ in the upper and lower surfaces of the $i$-th layer in portion $A B$ of un-cracked part of the beam. For this purpose, $n_{U}, \kappa_{1}, z_{1 n_{1}}, z_{1 i}, z_{1 i+1}, \sigma_{q_{i}}$ and $\sigma_{r_{i}}$ have to be replaced, respectively, with $n, \kappa_{2}, z_{2 n_{2}}, z_{2 i}, z_{2 i+1}, \sigma_{A B q_{i}}$ and $\sigma_{B D r_{i}}$, where $n$ is number of layers in the un-cracked beam part, $z_{2 i}$ and $z_{2 i+1}$ are, respectively, the coordinates of the upper and lower surfaces of the $i$-th layer. Also, equations (10), (11), (13) and (14) can be used to determine $\kappa_{3}, z_{3 n_{3}}, \sigma_{A B q_{i}}$ and $\sigma_{B D r_{i}}$ in portion $B D$ of the uncracked part of the beam. For this purpose, $n_{U}$ has to be replaced with $n$. Besides, the bending moment $M_{y_{1}}$ in (11) has to be found by the formula $F\left(l-x_{4} / 2\right)$.

The strain energy is written as:

$$
U=U_{C}+U_{A B}+U_{B D},
$$

where $U_{C}, U_{A B}$ and $U_{B D}$ are the strain energies, respectively, in the upper crack arm and portions $A B$ and $B D$ of the un-cracked part of the beam. The strain energy 
in the upper crack arm is expressed as:

$$
U_{C}=b \sum_{i=1}^{i=n_{U}} \int_{0}^{a} \int_{z_{1 i}}^{z_{1 i+1}} u_{0 C_{i}} d x_{4} d z_{1}
$$

where $u_{0 C_{i}}$ is the strain energy density in the $i$-th layer. It is known that the strain energy density is equal to the area enclosed by the stress-strain curve. Thus, by integrating the stress-strain relation (6), one derives:

$$
u_{0 C_{i}}=\frac{\sigma_{i}^{2}}{2 E_{i}}+\frac{\sigma_{i}^{\frac{1+m_{i}}{m_{i}}}}{\left(1+m_{i}\right) H_{i}^{\frac{1}{m_{i}}}} .
$$

The strain energies in portions $A B$ and $B D$ of the un-cracked part of the beam are written as:

$$
\begin{aligned}
& U_{A B}=b \sum_{i=1}^{i=n} \int_{a}^{l} \int_{z_{2 i}}^{z_{2 i+1}} u_{0 A B_{i}} d x_{4} d z_{2}, \\
& U_{B D}=b \sum_{i=1}^{i=n} \int_{l}^{2 l} \int_{z_{3 i}}^{z_{3 i+1}} u_{0 B D_{i}} d x_{4} d z_{3},
\end{aligned}
$$

where $u_{0 A B_{i}}$ and $u_{0 B D_{i}}$ are the strain energy densities in the $i$-th layer of portions $A B$ and $B D$ of the un-cracked beam part.

By substituting of (3), (15), (16), (18) and (19) in (2), one arrives at:

$$
G=\frac{F a}{2 b}\left[\kappa_{1}-\kappa_{2}\right]-\sum_{i=1}^{i=n_{U}} \int_{z_{1 i}}^{z_{1 i+1}} u_{0 C_{i}} d z_{1}+\sum_{i=1}^{i=n} \int_{z_{2 i}}^{z_{2 i+1}} u_{0 A B_{i}} d z_{2},
$$

where $\kappa_{1}, \kappa_{2}, u_{0 C_{i}}$ and $u_{0 A B_{i}}$ are obtained by (10), (11), (13), (14) and (17) at $x_{4}=a$. Since $\sigma_{i}$ can not be expressed as a function of $z_{1}$, formula (9) has to be substituted in the first integral in (20) and the integration has to be performed in boundaries from $\sigma_{q_{i}}$ to $\sigma_{r_{i}}$. Similarly, in the second integral in (20), the integration has to be performed in boundaries from $\sigma_{A B q_{i}}$ to $\sigma_{B D r_{i}}$. The result is: 
(21)

$$
\begin{aligned}
G=\frac{F a}{2 b}\left[\kappa_{1}-\kappa_{2}\right]-\frac{1}{\kappa_{1}} \sum_{i=1}^{i=n_{U}}\left[\left(\sigma_{r_{i}}^{3}-\sigma_{q_{i}}^{3}\right) \frac{1}{6 E_{i}^{2}}\right. \\
+\left(\sigma_{r_{i}}^{\frac{1+2 m_{i}}{m_{i}}}-\sigma_{q_{i}}^{\frac{1+2 m_{i}}{m_{i}}}\right) \frac{3 m_{i}+1}{2\left(1+2 m_{i}\right)\left(1+m_{i}\right) E_{i} H_{i}^{\frac{1}{m_{i}}}} \\
+\left(\sigma_{r_{i}}^{\frac{2+m_{i}}{m_{i}}}-\sigma_{q_{i}}^{\frac{2+m_{i}}{m_{i}}}\right) \frac{1}{\left.\left(1+m_{i}\right)\left(2+m_{i}\right) H_{i}^{\frac{2}{m_{i}}}\right]} \\
+\frac{1}{\kappa_{2}} \sum_{i=1}^{i=n}\left[\left(\sigma_{A B r_{i}}^{3}-\sigma_{A B q_{i}}^{3}\right) \frac{1}{6 E_{i}^{2}}\right. \\
+\left(\sigma_{A B r_{i}}^{\frac{1+2 m_{i}}{m_{i}}}-\sigma_{A B q_{i}}^{\frac{1+2 m_{i}}{m_{i}}}\right) \frac{3 m_{i}+1}{2\left(1+2 m_{i}\right)\left(1+m_{i}\right) E_{i} H_{i}^{\frac{1}{m_{i}}}} \\
\left.+\left(\sigma_{A B r_{i}}^{\frac{2+m_{i}}{m_{i}}}-\sigma_{A B q_{i}}^{\frac{2+m_{i}}{m_{i}}}\right) \frac{1}{1+m_{i}} 2+m_{i} H_{i}^{\frac{2}{m_{i}}}\right]
\end{aligned}
$$

where $\sigma_{q_{i}}, \sigma_{r_{i}}, \sigma_{A B q_{i}}$ and $\sigma_{B D r_{i}}$ are determined from (10), (11), (13), (14) at $x_{4}=a$.

The solution to the strain energy release rate (21) is verified by using the fact that the strain energy release rate can be obtained also by differentiating the complementary strain energy with respect to the delamination crack area. Thus, the strain energy release rate is written as:

$$
G=\frac{d U^{*}}{b d a},
$$

where $d a$ is an elementary increase of the delamination crack length. The complementary strain energy $U^{*}$ that is involved in (22) is found by (15), (16), (18) and (19). For this purpose, the strain energy densities $u_{0 C_{i}}, u_{0 A B_{i}}$ and $u_{0 B D_{i}}$ are replaced, respectively, with the complementary strain energy densities $u_{0 C_{i}}^{*}, u_{0 A B_{i}}^{*}$ and $u_{0 B D_{i}}^{*}$. In principle, the complementary strain energy density is equal to the area that complements the area enclosed by the stress-strain curve to a rectangle. Thus, the complementary strain energy density $u_{0 C_{i}}^{*}$ can be written as:

$$
u_{0 C_{i}}^{*}=\sigma_{i} \varepsilon-u_{0 C_{i}} .
$$

By combining of (6) and (17), one derives:

$$
u_{0 C_{i}}^{*}=\frac{\sigma_{i}^{2}}{2 E_{i}}+\frac{m_{i} \sigma_{i}^{\frac{1+m_{i}}{m_{i}}}}{\left(1+m_{i}\right) H_{i}^{\frac{1}{m_{i}}}} .
$$


It should be mentioned that the strain energy release rates obtained by substituting of $U^{*}$ in (22) are exact match of the strain energy release rates determined by (21). This fact is a verification of the non-linear delamination analysis developed in the present paper.

\section{NumericAl RESUlTS}

A parametric investigation of the delamination behaviour is performed. For this purpose, calculations of the strain energy release rate are carried-out by applying formula (21). The results obtained are presented in non-dimensional form by using the formula $G_{N}=G /\left(E_{1} b\right)$. Two three-layered beam configurations (with a delamination between layers 2 and 3 (Fig. 3a) and between layers 1 and 2 (Fig. 3b)) are considered in order to evaluate the effect of delamination crack position along the beam height. The thickness of each layer is $t$. It is assumed that $b=0.020 \mathrm{~m}, t=0.002 \mathrm{~m}$, $l=0.190 \mathrm{~m}$ and $F=20 \mathrm{~N}$. The strain energy release rate in non-dimensional form is plotted against $H_{2} / H_{1}$ ratio in Fig. 4 (both three-layered beam configurations shown
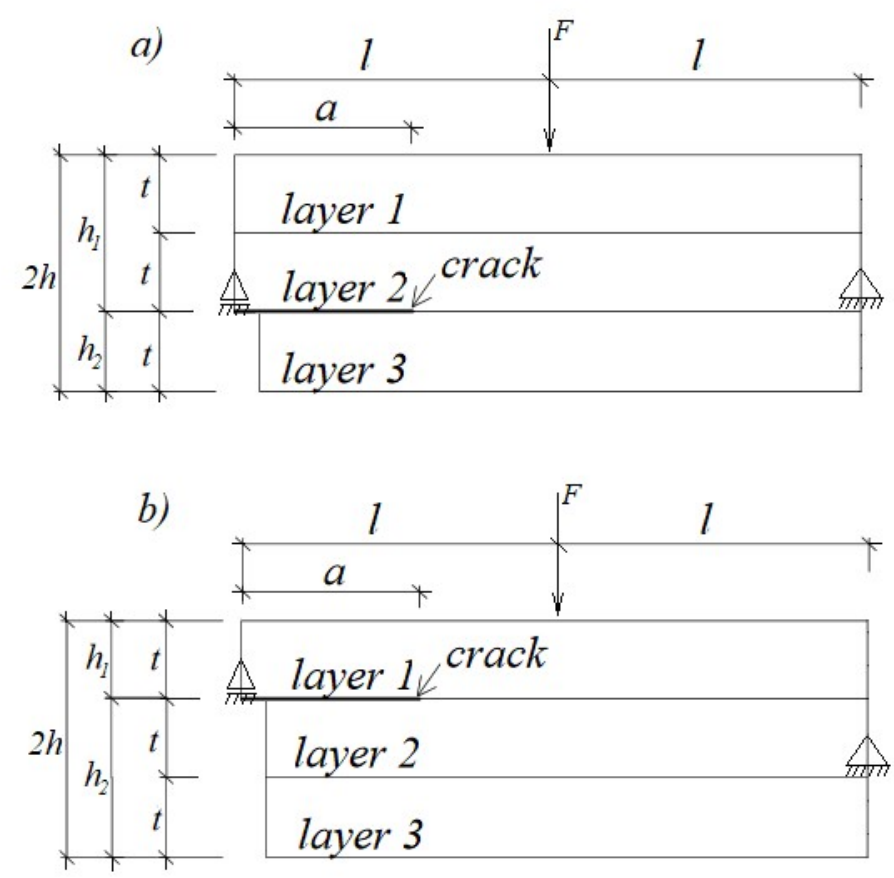

Fig. 3. Two three-layered beam configaratons with a delamination between: (a) layers 2 and 3 ; and (b) layers 1 and 2. 


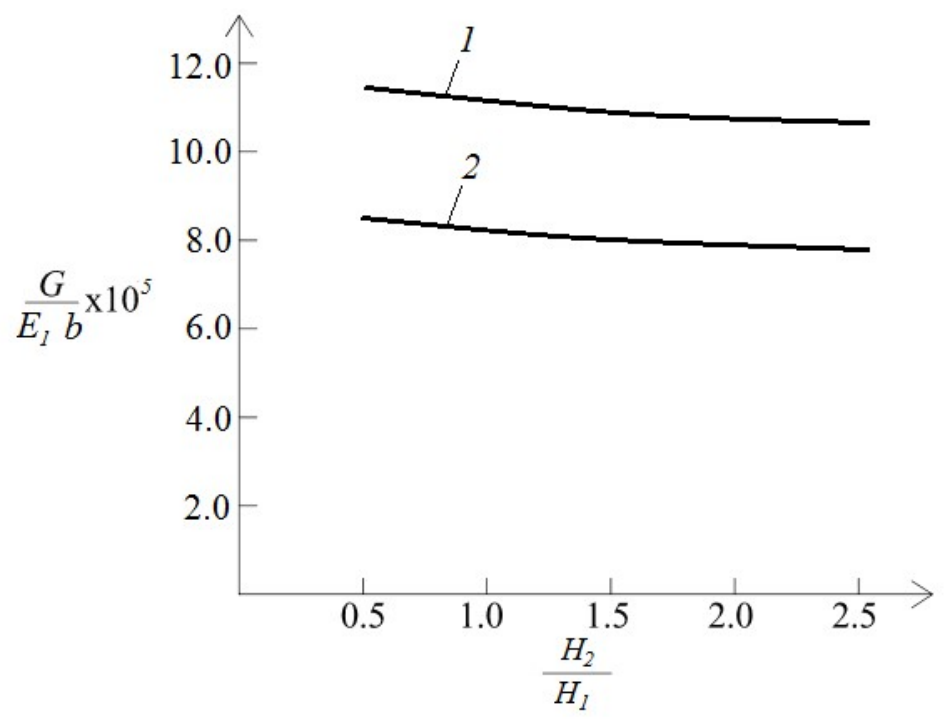

Fig. 4. The strain energy release rate in non-dimensional form plotted against $H_{2} / H_{1}$ ratio (curve 1 - for delamination between layers layers 1 and 2, curve 2 - for delamination between 2 and 3$)$.

in Fig. 3 are considered) assuming that $E_{2} / E_{1}=0.6, E_{3} / E_{1}=0.7, H_{1} / E_{1}=0.9$, $H_{3} / H_{1}=0.8, a / l=0.75$ and $m_{1}=m_{2}=m_{3}=0.8$. The curves in Fig. 4 indicate that the strain energy release rate decreases with increasing of $H_{2} / H_{1}$ ratio. It can also be observed in Fig. 4 that the strain energy release rate is higher when the delamination is between layers 1 and 2 (this behaviour is due to the fact that the upper crack arm stiffness is lower when the delamination is between layers 1 and 2).

The strain energy release rate in non-dimensional form is plotted against $E_{2} / E_{1}$ ratio in Fig. 5 (the three-layered beam with delamination between layers 1 and 2 (refer to Fig. 3b) is analyzed). One can observe in Fig. 5 that the strain energy release rate decreases with increasing of $E_{2} / E_{1}$ ratio. The effect of material non-linearity on the delamination behaviour is analyzed too. For this purpose, the strain energy release rate obtained assuming linear-elastic behaviour of the three-layered beam is also plotted in Fig. 5 for comparison. It should be noted that the linear-elastic solution for the strain energy release rate is derived by substituting of $H_{i} \rightarrow \infty$ in (10), (11), (13), (14) and (21). The curves in Fig. 5 show that the material non-linearity leads to increase of the strain energy release rate.

The influence of the delamination length on the fracture is also studied. For this purpose, the strain energy release rate in non-dimensional form is plotted against $H_{1} / E_{1}$ ratio in Fig. 6 at three $a / l$ ratios (the three-layered beam shown in Fig. $3 \mathrm{~b}$ is considered). It can be observed that the strain energy release rate increases with 


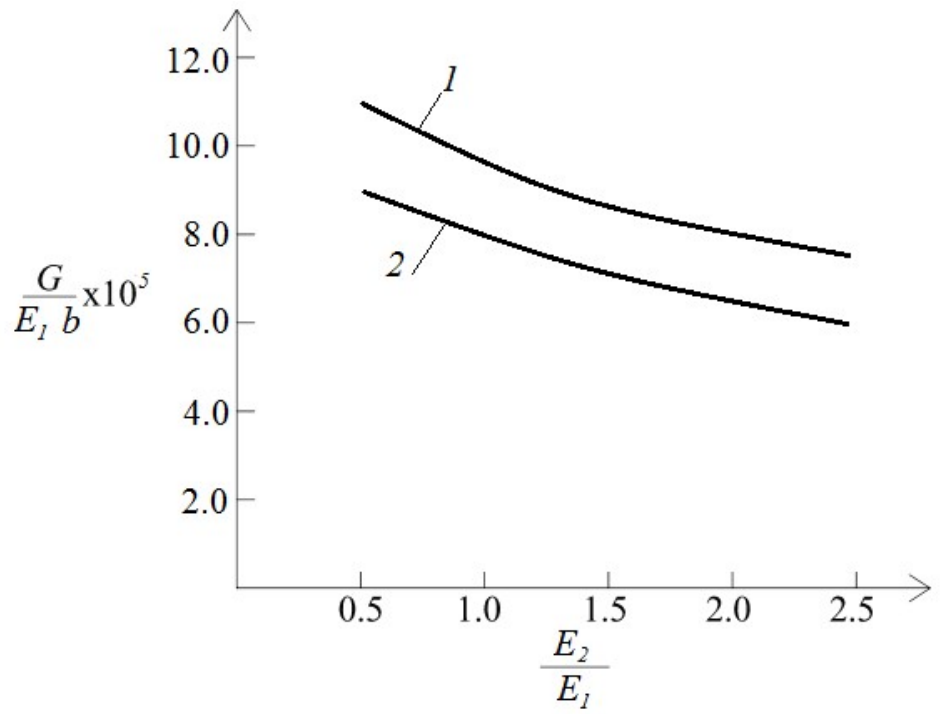

Fig. 5. The strain energy release rate in non-dimensional form plotted against $E_{2} / E_{1}$ ratio (curve 1 - at non-linear material behaviour, curve 2 - at linear-elastic behaviour).

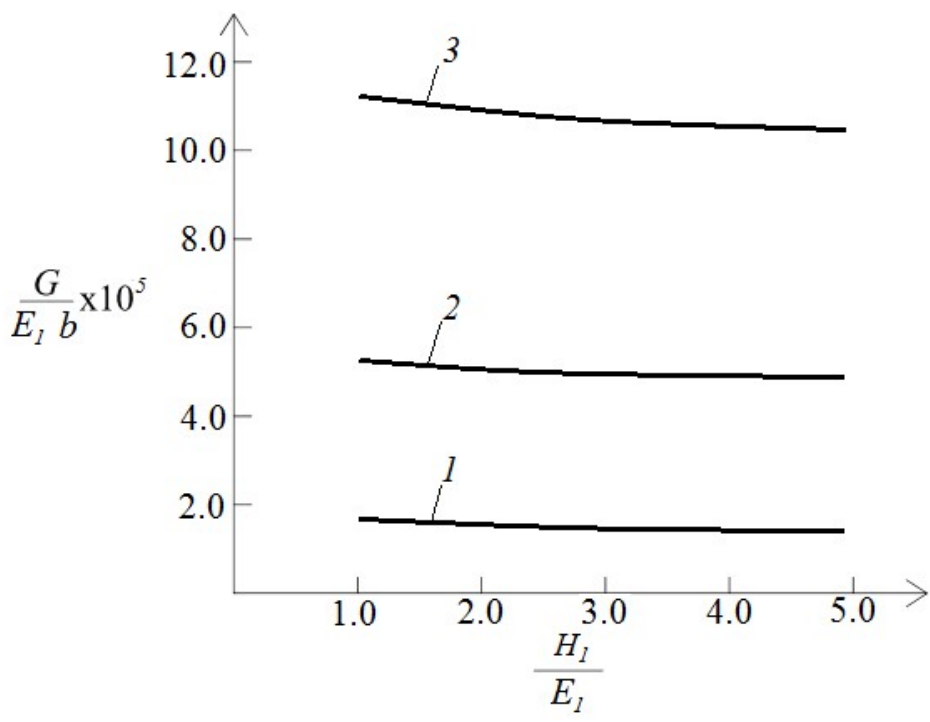

Fig. 6. The strain energy release rate in non-dimensional form plotted against $H_{1} / E_{1}$ ratio (curve $1-$ at $a / l=0.25$, curve $2-$ at $a / l=0.50$ and curve $3-$ at $a / l=0.75$ ).

increasing of $a / l$ ratio (Fig. 6). The increase of $H_{1} / E_{1}$ ratio leads to decrease of the strain energy release rate (Fig. 6). 


\section{CONCLUSIONS}

An analytical approach for analyzing the delamination behaviour of a multilayered three-point bending beam is developed assuming material non-linearity in each layer. The material non-linearity is treated by the Ramberg-Osgood equation. A solution for the strain energy release rate is derived by considering the eneregy balance. For verification, the strain eneergy release rate is determined also by analyzing the complementary strain energy. The solution derived can be applied for beams made of arbitrary number of longitudinal layers. Besides, the thickness and material properties can be different in each layer. Also, the delamination can be located arbitrary between layers. It should be specified that the solution derived holds for non-linear elastic material. However, the solution is applicable also for elastic-plastic behaviour if the beam undergoes active deformation, i.e. if the external load increases only. The solution is used to investigate the effects of delamination position along the beam height, delamination length and material non-linearity on the delamination behaviour. It is found that the strain energy release rate decreases with increasing of the thickness of the upper crack arm. The analysis revealed also that the strain eneergy release rate decreases with increasing of $H_{2} / H_{1}, E_{2} / E_{1}$ and $H_{1} / E_{1}$ ratios. It is found that the material non-linearity leads to increase of the strain energy release rate. The increase of the delamination length also leads to increase of the strain energy release rate.

The analytical approach developed in the present paper can be used as a tool for assessment of effects of material non-linearity in the structural design of multilayered three-point bending beam configurations with considering the delamination behaviour.

\section{ACKNOWLEDGMENTS}

Rizov gratefully acknowledges the financial support by DAAD for his research stay in Department of Technical Mechanics, Institute of Mechanics, Otto-von-GuerickeUniversity, Magdeburg, Germany.

\section{REFERENCES}

[1] M. Finot, S. SuReSh (1996) Small and large deformation of thick and thin-film multilayers: effect of layer geometry and compositonal gradients. Journal of the Mechanics and Physics of Solids $\mathbf{4 4}$ 683-721.

[2] J.S. Kim, K.W. PAIK, S.H. OH (1999) The Multilayer-Modified Stoney's Formula for Laminated Polymer Composites on a Silicon Substrate. Journal of Applied Physics $\mathbf{8 6}$ 5474-5479.

[3] L.B. FREUND (1995) The stress distribution and curvature of a general compositionally graded semiconductor layer. Journal of Crystal Growth 132 341-344.

[4] J. Malzbender (2003) Mechanical and thermal stresses in multilayered materials. Journal of Applied Physics 95 1780-1782. 
[5] J.-H. Yu, S. GuO, D.A. Gillard (2003) Bimaterial curvature measurements for CTE of adhesives: optimization and modeling. Journal of Adhesion Science and Technology 17 149-164.

[6] I. MARKov, D. Dinev (2005) Theoretical and experimental investigation of a beam strengthened by bonded composite strip. Reports of International Scientific Conference VSU'2005.

[7] R. MUŠÁLEK, O. KOVÁŘÍK, J. MATĚJíČEK (2010) In-situ observation of crack propagation in thermally sprayed coatings. Surface \& Coatings Technology 205 1807-1811.

[8] J.W. Hutchinson, Z. Suo (1992) Mixed mode cracking in layered materials. Advances in Applied Mechanics 64 804-810.

[9] W. Yang, C.F. Shin (1994) Fracture along an interlayer. International Journal of Solids and Structures 31 985-1002.

[10] B.D. DaVidson, V. Sundararaman (1996) A single-leg bending test for interfacial fracture toughness determination. International Journal of Fracture 78 193-210.

[11] N.W. KLingbeil, J.I. BeUth (1997) Interfacial fracture testing of deposited metal layers under four-point bending. Engineering Fracture Mechanics 56 113-126.

[12] J. Jiao, G.K. Gurumurthy, E.J. Kramer, Y. Sha, C.Y. Hui, P. Borgesen (1998) Measurement of interfacial fracture toughness under combined mechanical and thermal stress. Journal of Electronic Packaging 120 325-349.

[13] W.J. Cantwell, R. Scudamore, J. RatclifFe, P. Davies (1999) Interfacial fracture in sandwich laminates. Composites Science and Technology 59 2079-2085.

[14] D.T.S. Yeung, D.C.C. LAM, M.M.F. YuEN (2000) Specimen design for mixed mode interfacial fracture properties measurement in electronic packages. Journal of Electronic Packaging 122 67-72.

[15] F.G. Guadette, A.E. Giannapoulos, S. Suresh (2001) Interfacial cracks in layered materials subjected to a uniform temperature change. International Journal of Fracture 28 5620-5629.

[16] L. BRAndelli, R. MASSABo (2003) Free vibrations of delaminated beam-like structure with crack bridging. Composite Structures 61 129-142.

[17] J. WANG, C. ZHANG (2007) Delamination in elastic shear deformable circular layers. Composites Science and Technology 67 3323-3330.

[18] T. Yokozeki, T. Ogas AWARA, T. AOKI (2008) Correction method for evaluation of interfacial fracture toughness of DCB, ENF and MMB specimens with residual thermal stresses. Composites Science and Technology 68 760-767.

[19] S.-C. HER, W.-B. SU (2015) Interfacial fracture toughness of multilayered composite structures. Strength of Materials 47 24-32.

[20] V.I. Rizov (2018) Delamination in multi-layered functionally graded beams - an analytical study by using the Ramberg-Osgood equation. Structural Integrity and Life $\mathbf{1 8}$ 70-76.

[21] V.I. RIZOV (2018) Nonlinear delamination analysis of multilayered functionally graded circular shafts in torsion. Journal of Applied Mechanics and Technical Physics 591104 1110 . 